\title{
Randomized trial of combined triple therapy comprising two types of peginterferon with simeprevir in patients with hepatitis $\mathrm{C}$ virus genotype $1 b$
}

Akihiro Tamori, Kanako Yoshida, Osamu Kurai, Kiyohide Kioka, Hoang Hai, Ritsuzo Kozuka, Hiroyuki Motoyama, Etsushi Kawamura, Atsushi Hagihara, Sawako Uchida - Kobayashi, Hiroyasu Morikawa, Masaru Enomoto, Yoshiki Murakami, Norifumi Kawada

\begin{tabular}{|c|l|}
\hline Citation & Hepatology Research, 46(13); 1311-1320 \\
\hline Issue Date & 2016 -12 \\
\hline Type & Journal Article \\
\hline Textversion & Author \\
\hline $\begin{array}{c}\text { Supporting } \\
\text { Information }\end{array}$ & Supporting Information is available at https://doi.org/10.1111/hepr.12689 \\
\hline Rights & $\begin{array}{l}\text { This is the peer reviewed version of the following article: TAMORI, A., et al. (2016). } \\
\text { Randomized trial of combined triple therapy comprising two types of peginterferon } \\
\text { with simeprevir in patients with hepatitis C virus genotype 1b. Hepatology Research. } \\
\text { 46, 1311-1320., which has been published in final form at } \\
\text { https://doi.org/10.1111/hepr.12689 . This article may be used for non-commercial } \\
\text { purposes in accordance with Wiley Terms and Conditions for Self-Archiving. }\end{array}$ \\
\hline DOI & \begin{tabular}{l}
$10.1111 / h e p r .12689$ \\
\hline
\end{tabular}
\end{tabular}

Self-Archiving by Author(s)

Placed on: Osaka City University

TAMORI, A., et al. (2016). Randomized trial of combined triple therapy comprising two types of peginterferon with simeprevir in patients with hepatitis $\mathrm{C}$ virus genotype 1b. Hepatology Research. 46, 1311-1320. 
Randomized trial of combined triple therapy comprising two types of peginterferon with simeprevir in patients with HCV genotype $1 \mathrm{~b}$

Akihiro Tamori, ${ }^{1}$ Kanako Yoshida, ${ }^{1}$ Osamu Kurai, ${ }^{2}$ Kiyohide Kioka,${ }^{3}$ Hoang Hai, ${ }^{1}$ Ritsuzo Kozuka, ${ }^{1}$ Hiroyuki Motoyama, ${ }^{1}$ Etsushi Kawamura, ${ }^{1}$ Atsushi Hagihara, ${ }^{1}$ Sawako Uchida-Kobayashi, ${ }^{1}$ Hiroyasu Morikawa, ${ }^{1}$ Masaru Enomoto, ${ }^{1}$ Yoshiki Murakami, ${ }^{1},{ }^{4}$ and Norifumi Kawada ${ }^{1}$

${ }_{1}^{1}$ Department of Hepatology, Osaka City University Graduate School of Medicine, Osaka, Japan, ${ }^{2}$ Department of Gastroenterology, Osaka City Juso Hospital, Osaka Japan, and ${ }^{3}$ Department of Hepatology, Osaka City General Hospital, Osaka Japan

Key words: hepatitis $\mathrm{C}$ virus; peginterferon a2a; peginterferon a2b; ribavirin; sustained virologic response.

Short running title: RCT of SMV triple therapy 
Abbreviations: alanine aminotransferase; ALT, Hepatitis C virus; HCV, rapid virologic responders; RVR, end of treatment response; EOTR, non-virologic responders; NVR, inosine triphosphatase; ITPA, interleukin 28 B; IL28B, peginterferon; PEG-IFN, sustained virologic response; SVR.

\section{Conflicts of interest}

In the past year, Dr. Akihiro Tamori has received research funding from Chugai Pharmaceutical Co., Ltd. Dr. Norifumi Kawada has received research funding from MSD K.K. and Chugai Pharmaceutical Co., Ltd. and a lecturer's fee from Janssen Pharmaceutical K.K. The other authors declare that they have nothing to disclose regarding funding or conflict of interest with respect to the study reported in this manuscript.

Abstract; 245 words

Text; 3443 words

3 tables, 3 figures, and 1 supplemental figure

40 references 


\section{ABSTRACT}

Aim: Simeprevir (SMV) is a potent, macrocyclic hepatitis C virus (HCV) non-structural (NS)3/4A protease inhibitor. This prospective study compared the efficacy and safety of SMV in combination with peginterferon $\alpha-2 a+$ ribavirin, $(\mathrm{P} 2 \mathrm{aR})$ and with peginterferon $\mathrm{a}-2 \mathrm{~b}+$ ribavirin $(\mathrm{P} 2 \mathrm{bR})$, in Japanese patients with HCV genotype $1 b$ infection.

Methods: HCV genotype $1 \mathrm{~b}$ patients were randomly assigned to receive SMV (100 mg QD) with P2aR for 12 weeks, then P2aR alone for 12 or 36 weeks; or SMV (100 mg QD) with P2bR for 12 weeks, then P2bR alone for 12 or 36 weeks. The primary endpoint was a sustained virologic response 24 weeks after completing treatment (SVR24).

Results: In total, 151 patients were randomly assigned to the P2aR $(n=76)$ or P2bR group $(\mathrm{n}=75)$. Six patients dropped out. SVR24 was achieved in $55(75.3 \%)$ of $73 \mathrm{P} 2 \mathrm{aR}$ patients and $55(76.4 \%)$ of $72 \mathrm{P} 2 \mathrm{bR}$ patients. There was no difference in the rate of SVR24 between the two groups $(p=0.88)$. No differences in the proportion of patients who became HCV RNA-negative were detected between the P2aR and P2bR groups. The two groups had comparable numbers of adverse events, which led to the discontinuation of treatment in $9.6 \%$ and $8.3 \%$ of participants in the P2aR and P2bR groups, respectively.

Conclusions: Peginterferon $\alpha-2 a$ or $a-2 b$ in combination with SMV + ribavirin therapy showed identical antiviral effects in patients with chronic hepatitis C. Also, the incidence of adverse events was identical for both regimens. 


\section{INTRODUCTION}

Chronic hepatitis C (CHC) due to infection with hepatitis C virus (HCV) affects $\sim 170$ million people worldwide and is the most common cause of chronic liver disease. ${ }^{1}$ Of all HCV-infected individuals, 20-30\% eventually develop liver cirrhosis or hepatocellular carcinoma (HCC). Elimination of HCV is important for preventing $\mathrm{HCC}$, especially in aged patients with advanced fibrosis or CHC. ${ }^{2,3}$ Telaprevir (TVR), a first-generation inhibitor of the NS3/4A serine protease, was approved as a direct-acting antiviral (DAA) against CHC. 4, 5 Previous studies have reported significantly higher SVR rates in both treatment-naïve and treatment-experienced $\mathrm{CHC}$ subjects with $\mathrm{HCV}$ genotype 1 who were treated with TVR-combined triple therapy as compared to those treated with peginterferon (PEG-IFN) and ribavirin (RBV) combination therapy. ${ }^{4-7}$ However, severe anemia and other serious adverse events (SAEs) occur more frequently in patients with CHC undergoing TVR-combined triple therapy. 8, 9

Simeprevir (SMV) is a potent, macrocyclic NS3/4A protease inhibitor that exhibits pronounced antiviral activity in vitro and in vivo and has been approved as a combination therapy with PEG-IFN and RBV for patients with HCV genotype 1. ${ }^{10}$, ${ }^{11}$ In phase III trials, SMV combined triple therapy achieved a $85 \%$ sustained viral response (SVR) rate in treatment-naïve patients, and 90\% and 50\% SVR rates in previously relapsed patients and non-responders, respectively. ${ }^{12-14}$ In Japan, peginterferon $a-2 a$ and $a-2 b$ were approved for use in combination with SMV and RBV. To date, two types of PEG-IFN have been utilized in combination with RBV. PEG-IFN a-2a (40 kDa) comprises recombinant IFN a-2a covalently linked by a stable amide bond to a large, branched $40 \mathrm{kDa}$ PEG polymer to enhance its 
stability in vivo, and $180 \mu \mathrm{g}$ per week is administered, irrespective of patient body weight. In contrast, the PEG moiety in PEG-IFN a-2b (12kDa) is linked to histidine residues by urethane bonds. The PEG-IFN $\alpha-2 b$ dose is determined according to body weight. Some studies have reported that PEG-IFN a-2a therapy achieved a higher SVR rate than PEG-IFN $a-2 b$ therapy because PEG-IFN $\alpha-2 a$ was more stable after injection. ${ }^{15-17}$ However, AEs that necessitated dose reduction or treatment discontinuation were frequent during PEG-IFN a-2a therapy ${ }^{18}$ for low-body-weight patients; e.g., Japanese females. ${ }^{19}$ The type of PEG-IFN that has superior anti-HCV efficacy in patients with chronic liver disease remains controversial.

In addition, sufficient evidence of the efficacy and safety of the two types of PEG-IFN, in SMV combination therapy, is not available. Therefore, the present prospective study was designed and performed to compare the anti-HCV efficacy and safety of SMV, peginterferon $\alpha-2 \mathrm{a}$ and $\alpha-2 \mathrm{~b}$ triple therapy in $\mathrm{CHC}$ patients. 


\section{PATIENTS AND METHODS}

\section{Patients}

This study enrolled adult patients, aged $20-80$ years, who were chronically infected with HCV genotype $1 \mathrm{~b}$ and had a serum HCV RNA viral load $>3$ log copies/mL at screening. Additional inclusion criteria were platelet count $\geq$ $80,000 / \mathrm{mm}^{3}$, and hemoglobin $\geq 10 \mathrm{~g} / \mathrm{dL}$. Patients were excluded if they had a medical contraindication to PEG-IFN or RBV, a history of DAA use, a history of drug use, documented cirrhosis, or evidence of other significant liver diseases including hepatitis B-related diseases, autoimmune liver diseases, alcohol liver disease and HCC. No patient showed evidence of human immunodeficiency virus infection. This study was conducted according to the guidelines of the 1975 Declaration of Helsinki (2004 version), and written informed consent was obtained from all patients prior to treatment. The study protocol was approved by the Ethics Committee of each hospital and by the Ethics Committee of Osaka City University Graduate School of Medicine (No. 2677). The trial was registered in UMIN (No. 000012384).

\section{Study design}

Patients from three hospitals were evaluated in a randomized, two-arm study. The efficacy and safety of two types of PEG-IFNa were evaluated in patients receiving SMV (Sovriad ${ }^{\circledR}$; Janssen Pharmaceutical K.K., Tokyo, Japan) in combination with PEG-IFNa and body-weight-based RBV for 12 weeks, followed by PEG-IFNa and RBV for 12 weeks in treatment-naïve and relapsed patients, and for 36 weeks in previous non-responders. SMV was initiated at a dose of 100 mg per day. Before initiating treatment, 151 enrolled patients were allocated 
randomly to two groups [group P2aR: PEG-IFN a-2a (180 $\mu$ g/week Pegasys ${ }^{\circledR}$; Chugai Pharmaceutical Co. Ltd., Tokyo, Japan) and body-weight-based RBV (Copegus $^{\circledR}$, Chugai Pharmaceutical Co. Ltd.); group P2bR: PEG-IFN a-2b (1.5 $\mu \mathrm{g} / \mathrm{kg} /$ week Peg-Intron ${ }^{\circledR} ;$ MSD, Tokyo, Japan) and body-weight-based RBV (Rebetol $\left.\left.{ }^{\circledR}, \mathrm{MSD}\right)\right]$.

\section{Virologic evaluations}

HCV-RNA was determined using the TaqMan HCV assay $\left(\mathrm{COBAS}^{\circledR}{ }^{\circledR} \operatorname{TaqMan}^{\circledR}\right.$ HCV assay; Roche Molecular Diagnostics, Tokyo, Japan) with a lower limit of quantification of $15 \mathrm{IU} / \mathrm{mL}$ and an upper limit of quantification of $6.9 \times 10^{7} \mathrm{IU} / \mathrm{mL}$ (1.2-7.8 $\log \mathrm{IU} / \mathrm{mL})$. The HCV genotype was determined using an HCV genotype primer kit (Institute of Immunology Co., Ltd., Tokyo, Japan). Resistant-associated variants $(\mathrm{RAV})$ in the HCV NS3/4A gene were evaluated using the direct sequencing method according to a protocol reported previously. ${ }^{20}$ HCV RNA was extracted from $100 \mathrm{~mL}$ serum using the QIAmp Viral RNA Mini Kit (Qiagen, Hilden, Germany). Extracted RNA was reverse transcribed to complementary DNA using the Transcriptor First Strand cDNA Synthesis Kit (Roche Diagnostics K.K., Tokyo, Japan) with random hexamers. The NS3/4A region of the HCV genome was amplified using gene-specific forward (5'-ACACCGCGGCGTGTGGGGACAT-3'; nucleotides 3295-3316) and reverse (5'-GCTCTTGCCGCTGCCAGTGGGA-3'; nucleotides 4040-4019) primers. PCR products were purified using the QIAquick PCR Purification Kit (Qiagen, Hilden, Germany) and sequenced using the BigDye Terminator ver. 3.1 Cycle Sequencing Kit and an ABI 3730 xl Genetic Analyzer (Applied Biosystems, Foster City, CA, USA). Signals of $>10 \%$ of the baseline were considered positive. Resistance 
analysis focused on amino acid polymorphisms detected in patients who experienced failed treatment with HCV protease inhibitors, including SMV. These variants encompass amino acid residues V36, T54, Q80, R155, R156, and D168 within the NS3 protease domain.

A previous virologic response to IFN-based therapy was defined as follows: prior relapse showing undetectable HCV RNA at the end of treatment but detectable HCV RNA within 24 weeks after the end of treatment; and the re-appearance of HCV RNA at any time during treatment after a virologic response (breakthrough). Patients in whom HCV-RNA remained detectable during treatment were defined as non-responders.

\section{Endpoints}

The primary efficacy endpoint was the rate of SVR24, defined as undetectable HCV-RNA at 24 weeks after completion of treatment. Secondary virologic endpoints included the proportion of patients with rapid virologic response (RVR; undetectable serum HCV-RNA at week 4); complete early virologic response (EVR; undetectable serum HCV-RNA at week 12); end-of-treatment response (EOTR); and SVR12 (undetectable HCV-RNA at 12 weeks after completion of treatment). All methods of assessing treatment efficacy were defined according to previous guidelines. ${ }^{21}$ During the follow-up period, clinical, biochemical and quantitative serum HCV-RNA assessments were evaluated at 1-3-month intervals. Safety evaluations included adverse event reporting, laboratory test values, physical examinations, and vital sign assessments.

\section{SNP genotyping}

We examined the genetic polymorphisms in the interleukin 28 B (IL28B) and 
inosine triphosphatase (ITPA) genes in patients who consented to genetic analysis. 22, 23 Whole blood was collected from patients and centrifuged to separate the buffy coat. Genomic DNA was extracted from the buffy coat using a QIAamp ${ }^{\circledR}$ DNA Blood Midi Kit (Qiagen, Maryland, Germantown, MD, USA). Genetic polymorphisms in $I L 28 B$ rs8099917 and rs12979860 and ITPA rs1127354 were genotyped using the TaqMan SNP Genotyping Assay with the 7500 Fast Real-Time PCR System (Applied Biosystems, Foster City, CA, USA). All samples were also subjected to direct sequencing to confirm the genotype. The primers and procedures were used as described previously. ${ }^{22,23}$

\section{Statistical analysis}

The target enrollment was approximately 400 patients. With this sample size, differences of $>10 \%$ in the SVR rates between the two types of PEG-IFN would result in an $80 \%$ power to demonstrate that one of the PEG-IFNs was superior to the other PEG-IFN at an alpha level of 0.05 , as measured by the proportion of patients achieving SVR24.

Data analyses were conducted using the JMP software (ver. 9.0; SAS Institute, Cary, NC, USA). Differences between groups were evaluated by Wilcoxon's two-sample test for numerical variables or Fisher's exact test for categorical variables. Variables exhibiting $p$-values $<0.1$ in the univariate analysis were subjected to stepwise multivariate logistic regression analysis. In the two-tailed test, a $p$-value $<0.05$ was taken to indicate statistical significance. 


\section{RESULTS}

\section{Patients at baseline}

A total of 208 patients were screened for enrollment in this study, of whom 57 did not agree to participate; therefore, 151 patients were randomly allocated to the two groups and followed-up until SVR24 determination. Three patients treated with P2aR, and three treated with P2bR, dropped out of the protocol and so were excluded from the study. Finally, we compared efficacy and safety between 73 patients in the P2aR group and 72 patients in the P2bR group (Fig. 1). The characteristics of the patients in the P2aR and P2bR groups are listed in Table 1. The P2aR group comprised 36 females and 37 males, and the P2bR group had 49 females and 23 males $(p=0.02)$. The median age was 66 years (range: $30-77$ years) in the $\mathrm{P} 2 \mathrm{aR}$ group, and 64 years (range: $36-75$ years) in the $\mathrm{P} 2 \mathrm{bR}$ group ( $p$ $=0.11)$. The P2bR group had a predominance of female patients compared to the P2aR group. Previous IFN-based therapies were performed in 44 patients (57\%) in the P2aR group and 33 (43\%) in the P2bR group. The P2aR group had 27 relapsers and 17 non-responders, whereas the P2bR group had 19 relapsers and 14 non-responders $(p=0.74)$. In non-responders, elongation therapy with 36 weeks of PR was performed in five patients in the P2aR group, and four in the P2bR group. No significant differences in hematological and biochemical data were detected at baseline between the $\mathrm{P} 2 \mathrm{aR}$ and $\mathrm{P} 2 \mathrm{bR}$ groups. The mean HCV-RNA level was $6.6 \pm 0.5 \log$ copies/mL in the P2aR group and $6.4 \pm 0.8 \mathrm{log}$ copies/mL in the P2bR group $(p=0.39)$. RAVs in the HCV NS3/4A gene were detected in 8 (14\%) of 56 patients in the P2aR group: T54S and Q80L, 4; Q80A, 1; Q80L, 2; and Q80L and R155Q, 1. RAVs were detected in 4 (7\%) of 56 patients in 
the P2bR group: T54T/N and Q80Q/E, 1; Q80L, 1; A156G, 1; and D168E, 1. No differences in the distribution of the IL28B and ITPA genotypes were detected between the P2aR and P2bR groups.

\section{Treatment responses in the P2aR and P2bR groups}

The RVR rate was 77\% (56/73) in the P2aR group and 85\% (61/72) in the P2bR group $(p=0.22)$. The EVR and EOT rates were $96 \%(69 / 72)$ and $96 \%(70 / 73)$ in the P2aR group and 97\% (67/69) and 94\% (68/72) in the P2bR group, respectively ( $p=$ 0.68 , and $p=0.68)$. The SVR24 rate was $75 \%(55 / 73)$ in the P2aR group and $76 \%$ (55/72) in the P2bR group ( $p=0.88)$. No significant difference in the treatment response rate was detected between the two groups (Fig. 2). Viral breakthrough occurred in three patients in the P2aR group and four patients in the P2bR group ( $p=0.68)$. Viral relapse occurred in 15 patients in the $\mathrm{P} 2 \mathrm{aR}$ group and 13 patients in the P2abR group $(p=0.74)$.

According to previous treatment history, enrolled patients were classified into the following three categories: treatment-naïve, relapse, and non-response. In treatment-naïve patients, the SVR24 rate was 76\% (22/29) in the P2aR group and $85 \%(33 / 39)$ in the P2bR group $(p=0.36)$. In relapsed patients and non-responders, the SVR24 rates were 89\% (24/27) and 53\% (9/17) in the P2aR group, and 84\% (16/19) and 43\% (6/14) in the P2bR group, respectively (Fig. 3A). No significant difference in the treatment response was detected between the two groups. In patients with $I L 28 B$ major, the SVR24 rate was $87 \%$ (46/53) in the P2aR group and 93\% (41/44) in the P2bR group (Fig. 3B, $p=0.30$ ). In patients with IL28B non-major, the SVR24 rate was 45\% (9/20) in the P2aR group and 50\% (14/28) in the P2bR group ( $p=0.73)$. 
In the P2aR group, the factors associated with SVR24 were female gender, low body weight, previous relapse, high platelet count, low total bilirubin at baseline, IL28B SNP, and RBV adherence (Table 2). Multivariate logistic regression analysis showed that $I L 28 B \operatorname{SNP}(p<0.001), \mathrm{RBV}$ adherence $(p<0.001)$, and total bilirubin $(p=0.01)$ were independently related to SVR24 in all 73 patients. In the P2bR group, the factors associated with SVR24 were previous relapse, IL28B SNP, and RBV adherence. IL28B SNP $(p<0.001)$ and RBV adherence $(p=0.001)$ were independently related to SVR24 in all 72 patients.

\section{Adverse events and treatment discontinuation in the P2aR and P2bR groups}

Treatment was discontinued for the following reasons in the P2aR group (one patient for each reason): pancytopenia, skin eruption, hyperthyroidism, high fever, hemorrhage in the fundus of the eye, HCC development, and self-discontinuation. In the P2bR group, treatment was discontinued due to anemia in two patients, skin eruption in one, hyperthyroidism in one, ALT elevation in one, and appetite loss in one patient. No difference in the incidence of adverse events was detected between the two groups (Table 3).

Hyperbilirubinemia (>2.0 mg/dl) occurred more frequently during SMV combination therapy in the P2bR group $(14 / 72 ; 19.4 \%)$ than in the P2aR group $(4 / 73 ; 5.5 \%),(p=0.02)$. Grade $2 \mathrm{AE}$ hyperbilirubinemia $(\geq 3.0 \mathrm{mg} / \mathrm{dL})$ occurred in the P2aR $(2 / 73 ; 2.7 \%)$ and the P2bR groups ( $3 / 72 ; 4.2 \%),(p=0.98)$. After SMV administration, total bilirubin levels recovered to $<2.0 \mathrm{mg} / \mathrm{dl}$ in all patients. ALT levels were increased twofold from baseline in $9(12.3 \%)$ of 73 patients in the P2aR group and in $5(6.9 \%)$ of 72 patients in the P2bR $(\mathrm{p}=0.27)$. The mean ALT level was significantly higher at 4 and 8 weeks of therapy in the P2aR group compared 
to the P2bR group (Supplemental Figure A). Neutrophil counts in peripheral blood were significantly decreased at 12 weeks and EOT in the P2aR group. During treatment, hemoglobin levels and platelet counts exhibited identical patterns in both groups (Supplemental Figure C, D and E). No patients in the present study died due to treatment-related adverse events.

\section{Treatment adherence in the P2aR and P2bR groups}

Among the patients treated for 24 weeks, the treatment adherence rate was $100 \%$ in patients that did not require adjustment due to $\mathrm{AE}$. The rate of PEG-IFN adherence was $87 \pm 17 \%$ in the P2aR group and $87 \pm 14 \%$ in the P2bR group ( $p=$ 0.84). Also, the rate of RBV adherence was $88 \pm 16 \%$ in the P2aR group and $84 \pm$ $18 \%$ in the $\mathrm{P} 2 \mathrm{bR}$ group $(p=0.21)$. 


\section{DISCUSSION}

Several studies have suggested that the antiviral effect of PEG-IFNa-2a was superior to that of PEG-IFNa-2b in combination with only RBV. ${ }^{15-17}$ However, other studies reported that both PEG-IFNs achieved the same effect.18, 24, 25 To the best of our knowledge, few studies of both PEG-IFNs in combination with DAA plus RBV have been published. In the QUEST-2 study of combination therapy with SMV plus RBV, which included patients with HCV genotypes 1a and 1b, the SVR12 rate was $88 \%$ in the PEG-IFNa-2a group and $78 \%$ in the PEG-IFNa-2b group. ${ }^{26}$ In Japanese Phase III trials of SMV triple therapies, ${ }^{12-14}$ PEG-IFNa-2a was used in the CONCERTO-1, 2, and 3 studies, while PEG-IFNa-2b was used in the CONCERTO-4 study. In untreated and relapsed patients, the SVR24 rate was higher in the PEG-IFNa-2b treatment (91.7\%, 96.6\%) group compared to the PEG-IFNa-2a treatment group (88.6\%, 89.8\%). Conversely, non-responders had SVR24 rates higher in the PEG-IFNa-2a treatment group (44.7\%) compared to the PEG-IFNa-2b treatment group (38.5\%). In the present study the RVR, EOT, and SVR24 rates were not significantly different between the P2aR and P2bR groups in non-cirrhotic Japanese patients with HCV genotype 1b. Both the P2aR and P2bR groups exhibited similar SVR24 rates in treatment-naïve, relapsed, and non-responding patients. In both groups, IL28B genotype, RBV adherence, and previous response to IFN were associated with SVR24. Although addition of DAA to PEG-IFN improved the SVR rate, the most reliable predictive factors for SMV triple therapy were identical to those for combination therapy with PEG-IFN plus RBV. 22, 27, 28 In the P2aR group, female gender, low body weight, high platelet 
count, and low total bilirubin at baseline were also associated with SVR24; the reason why these factors were related to SVR in only the P2aR group is unclear. The PEG-IFNa-2a dose was fixed at $180 \mu \mathrm{g}$ per week even in patients with a body weight $>80 \mathrm{~kg}$. Only one of four patients with a body weight $>80 \mathrm{~kg}$ achieved an SVR24 in the P2aR group. It has been speculated that PEG-IFNa-2a may result in more adverse events in lower-body-weight patients compared to PEG-IFNa-2b if the dose is adjusted according to body weight. However, the incidence of treatment discontinuation was not different between the P2aR and P2bR groups, including in patients with a body weight $<50 \mathrm{~kg}$. The incidence of AEs without treatment discontinuation differed between the P2aR and P2bR groups. In the P2aR group, the incidence of ALT elevation and neutrophil count reduction was higher. Interestingly, two patients who had previously been treated with PEG-IFNa-2b + RBV had elevated ALT levels (>200 IU/L) when SMV was combined with PEG-IFNa-2a + RBV. These AEs should be taken into consideration in patients treated with SMV in combination with P2aR. Total bilirubin significantly increased during the SMV combined phase, in particular in the P2bR group (Table 3, and Supplemental Figure B). It was reported that such bilirubinemia is associated with SMV, not with PEG-IFN. When considering the fluctuations in total bilirubin during the first 12 weeks of SMV triple therapy, several factors could have contributed to hyperbilirubinemia, including the baseline bilirubin concentration, hemolysis due to RBV, and the patient's genetic background. ${ }^{29,} 30$ However, to the best of our knowledge, no study has indicated that increases in total bilirubin levels are due to the type of PEG-IFN administered. 
In phase III clinical trials in Japan, SMV combined triple therapy achieved an 85\% SVR rate in treatment-naïve patients, and a 95\% SVR rate in previously relapsed patients. ${ }^{12-14}$ Compared with those data, we found a lower SVR 24 rate in patients treated with SMV combined therapy. One of the reasons for this result was lower adherence to the treatment regimen in our cohort. Several adverse effects related to IFN occurred, in particular in older patients. Eleven patients could not continue the SMV combined therapy in our study. A recent study reported that IFN-free DAA therapy showed greater safety compared to IFN-based therapy. ${ }^{31-33}$ The HCV treatment guidelines of the American Association for the Study of Liver Diseases (AASLD), ${ }^{34}$ European Association for the Study of the Liver (EASL), ${ }^{35}$ and Japan Society of Hepatology (JSH) recommend that DAA combination regimens are the first line for anti-HCV therapy. However, multi-resistant HCV may emerge in patients who fail to respond to DAA therapy; IFN-based therapy may be effective in such patients. In addition, there are several reports showing that IFN suppresses the development of HCC, even when HCV infections continue. ${ }^{36-38}$ Thus, it is necessary to compare the long-term outcomes of patients treated with IFN-based therapies to patients treated with DAA.

The present study had several limitations. First, patients could not be enrolled to the primary planning number (UMIN No. 000012384). Since April 2015, no patients have been started on combined therapy with IFN in our hospital. Consequently, 151 random patients with SMV undergoing triple therapy were assessed. Our analyses revealed that SVR24 was achieved in 55 patients each in 
the P2aR (75.3\%) and P2bR (76.4\%) groups. There was only a 1\% difference in the SVR rates between the two types of PEG-IFN. It is unlikely that these results would be improved by enrolling 400 patients in the study. In addition, ethically, it is difficult to initiate SMV combined triple therapy in patients recommended to undergo IFN-free therapy. Therefore, we decided to halt the recruitment of patients to this study. Second, the gender distribution and history of IFN therapy were different between the two PEG-IFN groups. However, there was no significant difference in the number of non-responders between the two groups. Finally, we screened for RAVs in NS3/4 A before treatment in only $112(77 \%)$ of 145 treated patients. Baseline Q80K RAV in HCV genotype 1a has been reported to be related to failure of SMV with IFN and RBV therapy. ${ }^{26,39,40}$ However, RAV without D168 was not associated with the application of SVR triple therapy in patients with HCV genotype 1b. ${ }^{26,39,40}$ Taken together, our findings suggest that two types of PEG-IFNs in combination SMV triple therapy achieved an identical SVR rate in Japanese non-cirrhotic patients with HCV genotype 1b. Furthermore, the tolerability and safety of both PEG-IFNs were identical. 


\section{Acknowledgments}

We would like to thank Ms. Yoko Yasuhara, Ms. Tomoko Hirano and Ms. Sanae

Deguchi for collecting the data.

The English in this document has been checked by at least two professional editors, both native speakers of English. For a certificate, please see:

http://www.textcheck.com/certificate/ubszJr 
References

1. Pearlman BL. Protease inhibitors for the treatment of chronic hepatitis C genotype-1 infection: the new standard of care. Lancet Infect Dis. 2012; 12: 717-728.

2. Imai Y, Tamura S, Tanaka H, et al. Reduced risk of hepatocellular carcinoma after interferon therapy in aged patients with chronic hepatitis $\mathrm{C}$ is limited to sustained virological responders. J Viral Hepat. 2010; 17: 185-191.

3. Asahina Y, Tsuchiya K, Tamaki N, et al. Effect of aging on risk for hepatocellular carcinoma in chronic hepatitis $\mathrm{C}$ virus infection. Hepatology 2010; 52: 518-527.

4. Hézode C, Forestier N, Dusheiko G, et al. Telaprevir and peginterferon with or without ribavirin for chronic HCV infection. N Engl J Med. 2009; 360: 18391850 .

5. McHutchison JG, Manns MP, Muir AJ, et al. Telaprevir for previously treated chronic HCV infection. N Engl J Med. 2010; 362: 1292-1303.

6. Jacobson IM, McHutchison JG, Dusheiko G, et al. ADVANCE Study Team. Telaprevir for previously untreated chronic hepatitis $\mathrm{C}$ virus infection. $N$ Engl $J$ Med. 2011; 364: 2405-2416.

7. Zeuzem S, Andreone P, Pol S, et al. Telaprevir for retreatment of HCV infection. N Engl J Med. 2011; 364: 2417-2428.

8. Ogawa E, Furusyo N, Nakamuta M, et al. Clinical milestones for the prediction of severe anemia by chronic hepatitis $\mathrm{C}$ patients receiving telaprevir-based triple therapy. J Hepatol. 2013; 59: 667-674.

9. Tamori A, Kioka K, Sakaguchi H, et al. Effects on anemia of drug adjustment in patients with chronic hepatitis C during telaprevir-combined therapy. Ann Hepatol. 2015; 14: 28-35.

10. Lin TI, Lenz O, Fanning G, et al. In vitro activity and preclinical profile of TMC435350, a potent hepatitis C virus protease inhibitor. Antimicrob Agents Chemother. 2009; 53: 1377-1385.

11. M.W. Fried, M. Buti, G.J. Dore, et al. Once-daily simeprevir (TMC435) with pegylated interferon and ribavirin in treatment-naive genotype- 1 hepatitis $\mathrm{C}$ : The randomized PILLAR study. Hepatology. 2013; 58: 1918-1929. 
12. Hayashi N, Izumi N, Kumada H, et al. Simeprevir with peginterferon/ribavirin for treatment-naïve hepatitis $\mathrm{C}$ genotype 1 patients in Japan: CONCERTO-1, a phase III trial. J Hepatol. 2014; 61(2): 219-227.

13. Izumi N, Hayashi N, Kumada H, et al. Once-daily simeprevir with peginterferon and ribavirin for treatment-experienced HCV genotype 1-infected patients in Japan: the CONCERTO-2 and CONCERTO-3 studies. $J$ Gastroenterol. 2014; 49: 941-953.

14. Kumada H, Hayashi N, Izumi N, et al. Simeprevir (TMC435) once daily with peginterferon- $\alpha-2 b$ and ribavirin in patients with genotype 1 hepatitis $C$ virus infection: The CONCERTO-4 study. Hepatol Res. 2015; 45: 501-513.

15. Ascione A, De Luca M, Tartaglione MT, et al. Peginterferon alfa-2a plus ribavirin is more effective than peginterferon alfa-2b plus ribavirin for treating chronic hepatitis C virus infection. Gastroenterology. 2010; 138: 116122.

16. Rumi MG, Aghemo A, Prati GM, et al. Randomized study of peginterferon-alpha2a plus ribavirin vs. peginterferon-alpha2b plus ribavirin in chronic hepatitis C. Gastroenterology. 2010; 138: 108-115.

17. Witthoeft T, Hueppe D, John C, et al. Efficacy and tolerability of peginterferon alfa-2a or alfa-2b plus ribavirin in the daily routine treatment of patients with chronic hepatitis C in Germany: the PRACTICE study. $J$ Viral Hepat. 2010; 17: 459-468.

18. Di Bisceglie AM, Ghalib RH, Hamzeh FM, Rustgi VK. Early virologic response after peginterferon alpha-2a plus ribavirin or peginterferon alpha-2b plus ribavirin treatment in patients with chronic hepatitis C. J Viral Hepat. 2007; 14: 721-729.

19. Sato I, Shimbo T, Kawasaki Y, Mizokami M, Masaki N. Efficacy and safety of interferon treatment in elderly patients with chronic hepatitis C in Japan: A retrospective study using the Japanese Interferon Database. Hepatol Res. $2015 ; 45: 827-838$.

20. Shepherd SJ, Abdelrahman T, MacLean AR, Thomson EC, Aitken C, Gunson RN. Prevalence of HCV NS3 pre-treatment resistance associated amino acid variants within a Scottish cohort. J Clin Virol. 2015; 65: 50-53.

21. AASLD and IDSA. Recommendations for Testing, Managing, and Treating Hepatitis C 2014, http://www.hcvguidelines.org

22. Tanaka Y, Nishida N, Sugiyama M, et al. Genome-wide association of IL28B 
with response to pegylated interferon-alpha and ribavirin therapy for chronic hepatitis C. Nat Genet. 2009; 41: 1105-1109.

23. Hai H, Tamori A, Enomoto M, et al. Relationship between inosine triphosphate genotype and outcome of extended therapy in hepatitis $\mathrm{C}$ virus patients with a late viral response to pegylated-interferon and ribavirin. $J$ Gastroenterol Hepatol. 2014; 29: 201-207.

24. McHutchison JG, Lawitz EJ, Shiffman ML, et al. Peginterferon alfa-2b or alfa-2a with ribavirin for treatment of hepatitis $\mathrm{C}$ infection. $N$ Engl $J$ Med. 2006; 361(6): 580-593.

25. Escudero A, Rodríguez F, Serra MA, Del Olmo JA, Montes F, Rodrigo JM. Pegylated alpha-interferon-2a plus ribavirin compared with pegylated alpha-interferon-2b plus ribavirin for initial treatment of chronic hepatitis $\mathrm{C}$ virus: prospective, non-randomized study. J Gastroenterol Hepatol. 2008; 23(6): 861-866.

26. Manns M, Marcellin P, Poordad F, et al. Simeprevir with pegylated interferon alfa $2 \mathrm{a}$ or $2 \mathrm{~b}$ plus ribavirin in treatment-naive patients with chronic hepatitis $\mathrm{C}$ virus genotype 1 infection (QUEST-2): a randomised, double-blind, placebo-controlled phase 3 trial. Lancet. 2014; 384: 414-426.

27. Akuta N, Suzuki F, Kawamura Y, et al. Predictive factors of early and sustained responses to peginterferon plus ribavirin combination therapy in Japanese patients infected with hepatitis $\mathrm{C}$ virus genotype $1 \mathrm{~b}$ : amino acid substitutions in the core region and low-density lipoprotein cholesterol levels. J Hepatol. 2007; 46: 403-410.

28. Ge D, Fellay J, Thompson AJ, et al. Genetic variation in IL28B predicts hepatitis C treatment-induced viral clearance. Nature. 2009; 461: 399-401.

29 Furihata T, Matsumoto S, Fu Z, et al. Different interaction profiles of direct-acting anti-hepatitis $\mathrm{C}$ virus agents with human organic anion transporting polypeptides. Antimicrob Agents Chemother. 2014; 58 : 4555-4564.

30 Tahata Y, Hiramatsu N, Oze T, et al. The impact of an inosine triphosphate pyrophosphatase genotype on bilirubin increase in chronic hepatitis $\mathrm{C}$ patients treated with simeprevir, pegylated interferon plus ribavirin. $J$ Gastroenterol. 2015; in press.

31. Afdhal N, Reddy KR, Nelson DR, et al. Ledipasvir and sofosbuvir for previously treated HCV genotype 1 infection. N Engl J Med. 2014; 370: 1483- 
1493.

32. Mizokami M, Yokosuka O, Takehara T, et al. Ledipasvir and sofosbuvir fixed-dose combination with and without ribavirin for 12 weeks in treatment-naive and previously treated Japanese patients with genotype 1 hepatitis C: an open-label, randomised, phase 3 trial. Lancet Infect Dis. 2015; 15:645-653.

33. Pearlman BL, Ehleben C, Perrys M. The combination of simeprevir and sofosbuvir is more effective than that of peginterferon, ribavirin, and sofosbuvir for patients with hepatitis C-related Child's class A cirrhosis. Gastroenterology. 2015 ; 148: 762-770.

34. HCV guidance: recommendations for testing, managing, and treating hepatitis C http://www.hcvguidelines.org/full-report-view.

35. EASL clinical practice guidelines; recommendations for treatment of hepatitis C 2015.

http://www.easl.eu/research/our-contributions/clinical-practice-guidelines/ detail/recommendations-on-treatment-of-hepatitis-c-2015.

36. Nishiguchi S, Shiomi S, Nakatani S, et al. Prevention of hepatocellular carcinoma in patients with chronic active hepatitis $\mathrm{C}$ and cirrhosis. Lancet 2001; 357: 196-197.

37. Lok AS, Everhart JE, Wright EC, et al. Maintenance peginterferon therapy and other factors associated with hepatocellular carcinoma in patients with advanced hepatitis C. Gastroenterology 2011; 140: 840-849.

38. Izumi N, Asahina Y, Kurosaki M, et al. Inhibition of hepatocellular carcinoma by $\mathrm{PegIFNa}-2 \mathrm{a}$ in patients with chronic hepatitis $\mathrm{C}$ : a nationwide multicenter cooperative study. J Gastroenterol. 2013; 48: 382-390.

39. Jacobson IM, Dore GJ, Foster GR, et al. Simeprevir (TMC435) with pegylated interferon alfa 2 a plus ribavirin in treatment-naive patients with chronic hepatitis $\mathrm{C}$ virus genotype 1 infection (QUEST-1): a phase 3, randomised, double-blind, placebo-controlled trial. Lancet. 2014; 384: 403-413.

40. Lenz O, Verbinnen T, Fevery B, et al. Virology analyses of HCV isolates from genotype 1-infected patients treated with simeprevir plus peginterferon/ribavirin in Phase IIb/III studies. J Hepatol. 2015; 62: 10081014. 
Table 1. Baseline characteristics of patients with chronic hepatitis $\mathrm{C}$ virus infection allocated to the PEG-IFNa-2a (P2aR) and PEG-IFNa-2b (P2bR) groups.

\begin{tabular}{|c|c|c|c|}
\hline & P2aR group & P2bR group & $p$-value \\
\hline Number of patients & 73 & 72 & \\
\hline Male/Female & $37 / 36$ & $23 / 49$ & 0.02 \\
\hline Median age (range) years & $66(30-77)$ & $64(36-75)$ & 0.11 \\
\hline Body weight (kg) & $58(41-94)$ & $56(43-86)$ & 0.18 \\
\hline Previous IFN therapy +/- & $44 / 29$ & $33 / 39$ & 0.08 \\
\hline (relapser/non־responder) & $27 / 17$ & $19 / 14$ & 0.74 \\
\hline $\begin{array}{l}\text { Fibrosis stage } \\
\text { (F0/F1/F2/F3/ND) }\end{array}$ & 0/22/17/4/30 & $1 / 20 / 20 / 9 / 22$ & 0.43 \\
\hline $\begin{array}{l}\text { IL28B genotype } \\
\text { (major/non-major) }\end{array}$ & $53 / 20$ & $44 / 28$ & 0.14 \\
\hline $\begin{array}{l}\text { ITPA genotype } \\
\text { (major/non-major) }\end{array}$ & $51 / 22$ & $47 / 24$ & 0.68 \\
\hline $\begin{array}{l}\text { HCV RNA (Log } \\
\text { copies/mL) }\end{array}$ & $6.6 \pm 0.5$ & $6.4 \pm 0.8$ & 0.39 \\
\hline NS3/4A RAVs +/-/ND & $8 / 48 / 17$ & $4 / 52 / 16$ & 0.36 \\
\hline $\mathrm{WBC}(/ \mathrm{mL})$ & $4881 \pm 1463$ & $4721 \pm 1377$ & 0.44 \\
\hline Neutrophil (/mL) & $2689 \pm 736$ & $2616 \pm 686$ & 0.39 \\
\hline $\mathrm{Hb}(\mathrm{g} / \mathrm{dL})$ & $13.9 \pm 1.3$ & $13.6 \pm 1.3$ & 0.12 \\
\hline Plt (x104/mL) & $16.7 \pm 5.3$ & $15.6 \pm 5.3$ & 0.24 \\
\hline T. Bil (mg/dL) & $0.7 \pm 0.3$ & $0.7 \pm 0.3$ & 0.85 \\
\hline ALT (IU/L) & $50.6 \pm 38.2$ & $51.6 \pm 39.4$ & 0.97 \\
\hline g-GTP (IU/L) & $42.5 \pm 32.8$ & $54 \pm 61$ & 0.51 \\
\hline Cre $(\mathrm{mg} / \mathrm{L})$ & $0.73 \pm 0.14$ & $0.72 \pm 0.2$ & 0.33 \\
\hline
\end{tabular}

Abbreviations: IFN, interferon; HCV, hepatitis C virus; RAVs, resistant associated variants; WBC, white blood cell; Hb, hemoglobin; Plt, platelet; T. Bil, total bilirubin; ALT, alanine aminotransferase; $\gamma$-GTP, gamma-glutamyl transpeptidase; Cre, creatinine 
Table 2. Factors associated with SVR24 in the PEG-IFNa-2a (P2aR) and PEG-IFNa-2b (P2bR) groups.

\begin{tabular}{|c|c|c|c|}
\hline & \multicolumn{3}{|c|}{ PEG-IFN a2a } \\
\hline & SVR24 $(\mathrm{n}=55)$ & Non-SVR24 (n = 18) & $p$-value \\
\hline Male/Female & $26 / 29$ & $11 / 7$ & 0.03 \\
\hline Median age (range) years & $66(30-77)$ & $67(36-74)$ & 0.41 \\
\hline Body weight (kg) & $51.4(33.3-84)$ & $61.2(45.7-93.6)$ & 0.03 \\
\hline Previous IFN therapy +/- & $33 / 23$ & $11 / 7$ & 0.93 \\
\hline (relapser/non-responder) & $24 / 9$ & $3 / 8$ & $<0.01$ \\
\hline $\mathrm{WBC}(/ \mu \mathrm{L})$ & $4805 \pm 1441$ & $5112 \pm 1548$ & 0.53 \\
\hline Neutrophil $(/ \mu \mathrm{L})$ & $2654 \pm 732$ & $2794 \pm 760$ & 0.49 \\
\hline $\mathrm{Hb}(\mathrm{g} / \mathrm{dL})$ & $13.7 \pm 1.2$ & $14.4 \pm 1.3$ & 0.1 \\
\hline $\operatorname{Plt}\left(\mathrm{x} 10^{4} / \mu \mathrm{L}\right)$ & $17.4 \pm 5.4$ & $14.5 \pm 4.6$ & 0.03 \\
\hline T. Bil (mg/dL) & $0.6 \pm 0.3$ & $0.9 \pm 0.3$ & 0.02 \\
\hline ALT (IU/L) & $48.1 \pm 33.4$ & $59 \pm 51.7$ & 0.79 \\
\hline g-GTP (IU/L) & $40.0 \pm 30.6$ & $51.9 \pm 38.8$ & 0.12 \\
\hline Cre $(\mathrm{mg} / \mathrm{L})$ & $0.73 \pm 0.14$ & $0.72 \pm 0.15$ & 1 \\
\hline $\operatorname{AFP}(\mathrm{ng} / \mathrm{ml})$ & $6.4 \pm 7.7$ & $8.7 \pm 7.7$ & 0.15 \\
\hline $\begin{array}{l}\text { IL28B genotype } \\
\text { (major/non-major) }\end{array}$ & $46 / 9$ & $7 / 11$ & $<0.01$ \\
\hline $\begin{array}{l}\text { ITPA genotype } \\
\text { (major/non-major) }\end{array}$ & $41 / 14$ & $10 / 8$ & 0.22 \\
\hline $\begin{array}{l}\text { Fibrosis stage } \\
\text { (F0/F1/F2/F3) }\end{array}$ & $0 / 17 / 15 / 4$ & $0 / 5 / 2 / 9$ & 0.24 \\
\hline HCV RNA (Log copies/mL) & $6.6 \pm 0.5$ & $6.5 \pm 0.5$ & 0.4 \\
\hline $\mathrm{NS} 3 / 4 \mathrm{~A}$ RAVs +/-/ND & $6 / 37 / 12$ & $2 / 11 / 5$ & 0.89 \\
\hline PEG-IFN adherence (\%) & $84.4 \pm 18.5$ & $80.2 \pm 23.3$ & 0.62 \\
\hline RBV adherence (\%) & $87.4 \pm 19$ & $76.8 \pm 22.5$ & 0.04 \\
\hline
\end{tabular}

\section{Continue}




\begin{tabular}{|c|c|c|}
\hline \multicolumn{3}{|c|}{ PEG-IFN a2b } \\
\hline SVR24 (n = 55) & Non-SVR24 (n = 17) & $p$-value \\
\hline $19 / 36$ & $4 / 13$ & 0.73 \\
\hline $64(36-75)$ & $65(40-71)$ & 0.97 \\
\hline $54.7(43.7-86)$ & $58.9(45-72.3)$ & 0.27 \\
\hline $22 / 33$ & $11 / 6$ & 0.07 \\
\hline $16 / 6$ & $3 / 8$ & 0.01 \\
\hline $4797 \pm 1471$ & $4475 \pm 1011$ & 0.74 \\
\hline $2647 \pm 730$ & $2520 \pm 528$ & 0.54 \\
\hline $13.5 \pm 1.3$ & $13.7 \pm 1.1$ & 0.61 \\
\hline $15.9 \pm 5.7$ & $14.8 \pm 4.5$ & 0.39 \\
\hline $0.7 \pm 0.3$ & $0.8 \pm 0.3$ & 0.27 \\
\hline $47.5 \pm 36.7$ & $64.5 \pm 45.4$ & 0.08 \\
\hline $53.9 \pm 67.8$ & $54.1 \pm 34$ & 0.21 \\
\hline $0.72 \pm 0.19$ & $0.7 \pm 0.25$ & 0.39 \\
\hline $8.4 \pm 11.5$ & $15.9 \pm 22.4$ & 0.32 \\
\hline $41 / 14$ & $3 / 14$ & $<0.01$ \\
\hline $35 / 20$ & $12 / 4$ & 0.23 \\
\hline $1 / 15 / 16 / 8$ & $0 / 5 / 4 / 1$ & 0.57 \\
\hline $6.3 \pm 0.8$ & $6.7 \pm 0.5$ & 0.2 \\
\hline $4 / 40 / 11$ & $0 / 12 / 5$ & 0.28 \\
\hline $87 \pm 14$ & $72 \pm 28$ & 0.08 \\
\hline $85 \pm 18$ & $66 \pm 27$ & $<0.01$ \\
\hline
\end{tabular}

Abbreviations: SVR24, sustained virologicl response 24 weeks after completing treatment; IFN, interferon; WBC, white blood cell; Hb, hemoglobin; Plt, platelet; T. Bil, total bilirubin; ALT, alanine aminotransferase; g-GTP, g-glutamyl transpeptidase; Cre, creatinine; AFP, alpha-fetoprotein; HCV, hepatitis C virus; RAV, resistant associated variants; PEG-IFN, peginterferon; RBV, ribavirin 
Table 3. Adverse events with and without treatment discontinuation in the PEG-IFNa-2a (P2aR) and PEG-IFNa-2b (P2bR) groups.

\begin{tabular}{rccc}
\hline & $\mathrm{P} 2 \mathrm{aR}(\mathrm{n}=73)$ & $\mathrm{P} 2 \mathrm{bR}(\mathrm{n}=72)$ & $p$-value \\
\hline Discontinuation due to AE & $6(8.2 \%)$ & $6(8.3 \%)$ & 0.98 \\
Pancytopenia (anemia) & 1 & 2 & \\
Skin eruption & 1 & 1 \\
Hyperthyroidism & 1 & 1 \\
Fever & 1 & 0 \\
Hemorrhage in eye fundus & 1 & 0 \\
Development of HCC & 1 & 1 \\
ALT elevation & 0 & 1
\end{tabular}

$\mathrm{AE}$ in clinical data without discontinuation

$\begin{array}{rccc}\text { Total bilirubin }>2.0 \mathrm{mg} / \mathrm{dL} & 4 & 14 & 0.02 \\ \text { Total bilirubin }>3.0 \mathrm{mg} / \mathrm{dL} & 2 & 3 & 0.98 \\ \text { ALT elevation }>2 \text { fold from baseline } & 9 & 5 & 0.41 \\ \text { Hemoglobin }<9.0 \mathrm{~g} / \mathrm{dL} & 7 & 8 & 0.98 \\ \text { Neutrophil }<750 / \mu \mathrm{L} & 15 & 8 & 0.17 \\ \text { Plt }<60,000 / \mu \mathrm{L} & 15 & 14 & 0.97 \\ & & & 0.89 \\ \text { (1) } & 83 \pm 20 & 84 \pm 19 & 0.28\end{array}$

Adherence of RBV (\%)

$84 \pm 20$

0.28

AEs, adverse events; HCC, hepatocellular carcinoma; ALT, alanine aminotransferase; Hb, hemoglobin; Plt, platelet 


\section{Figure Legends}

Figure 1.

Trial profile.

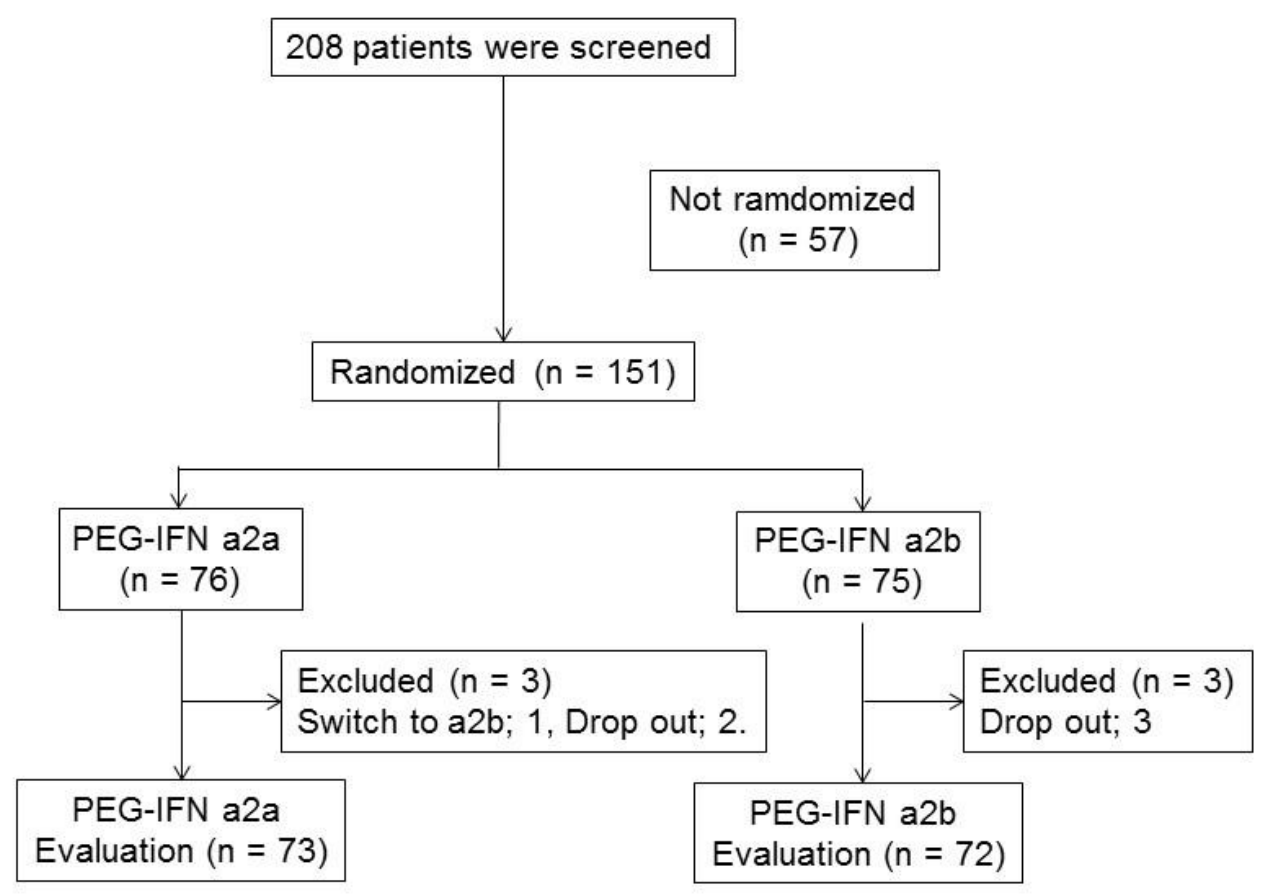


Figure 2.

Rapid virologic response (RVR), early virologic response (EVR), end-of-treatment response (EOTR), sustained virologic response 12 weeks after completing treatment (SVR12), and sustained virologic response 24 weeks after completing treatment (SVR24) in the PEG-IFNa-2a (P2aR) and PEG-IFNa-2b (P2bR) groups. The rates at all time points were not significantly different between the P2aR and P2bR groups.

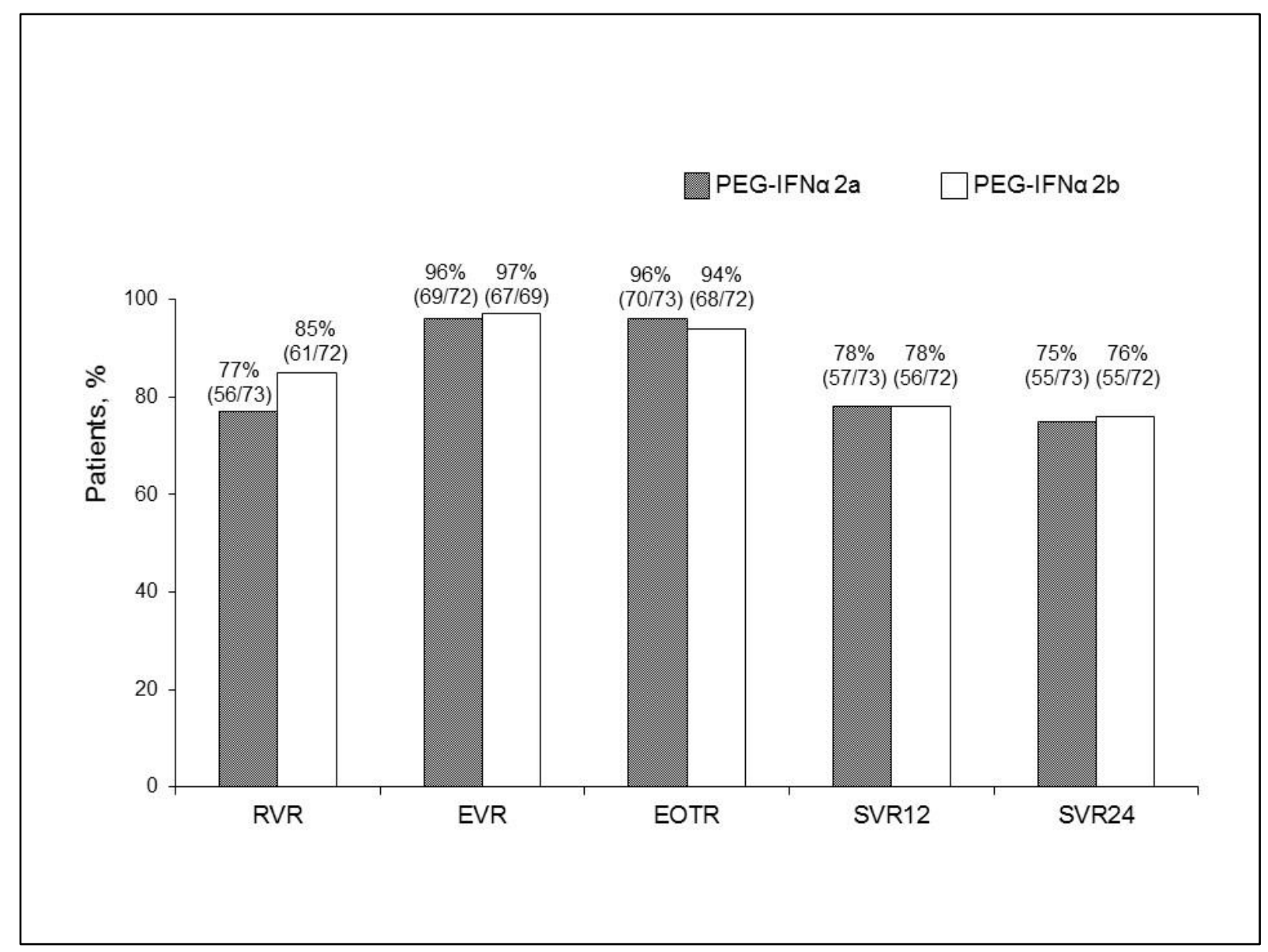


Figure 3.

A: Shown are the SVR24 rates in untreated, relapsed, and non-responding patients. No significant difference in the treatment response was observed between the P2aR and P2bR groups.

B: Shown are the SVR24 rates in patients with and without IL28B. No significant difference in the IL28B genotypes was detected between the P2aR and P2bR groups.

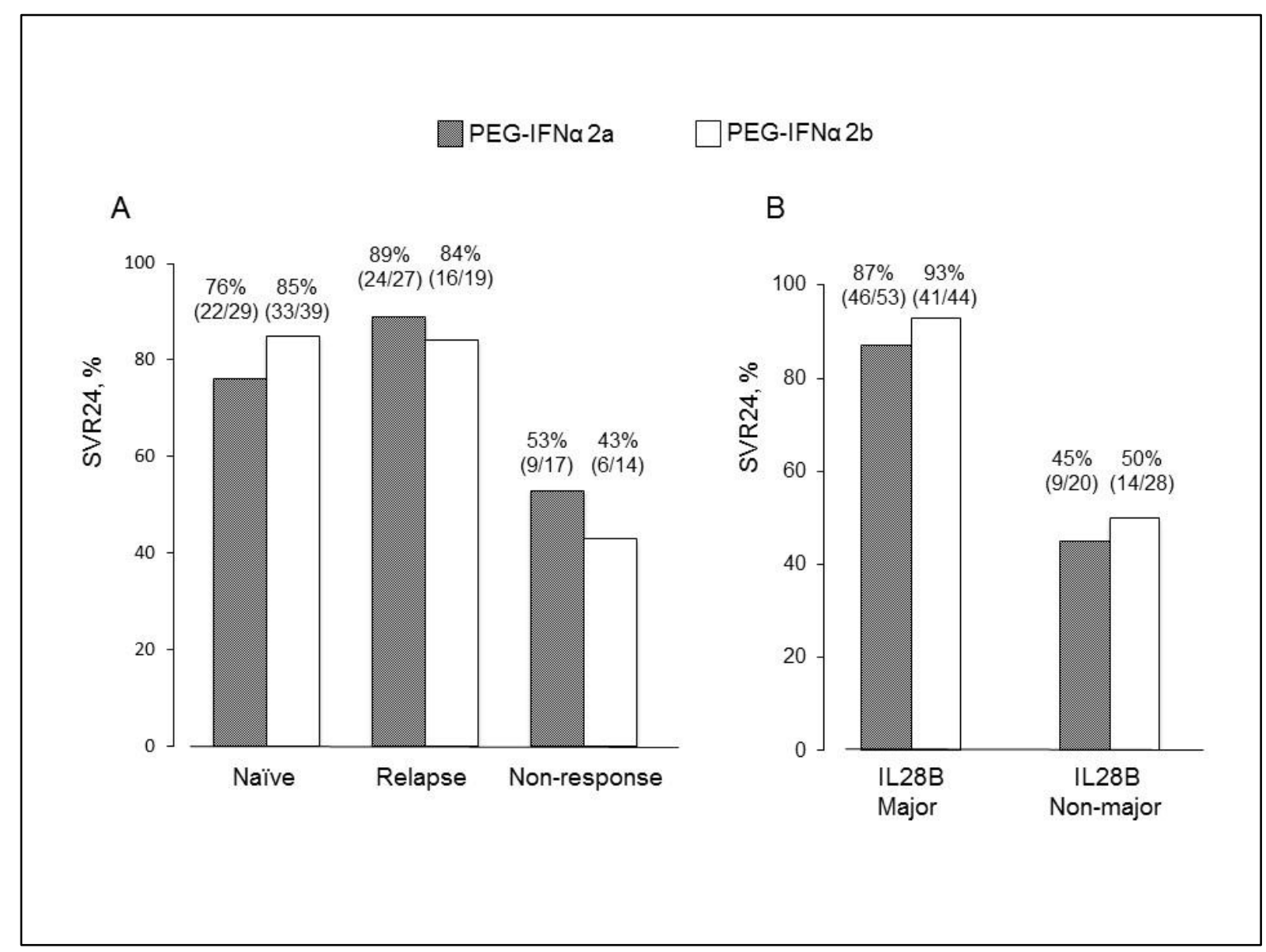


Supplementary Figure

Change from baseline laboratory observations during treatment period and

follow-up period: A alanine aminotransferase, B total bilirubin, C neutrophils, D platelets, E hemoglobin

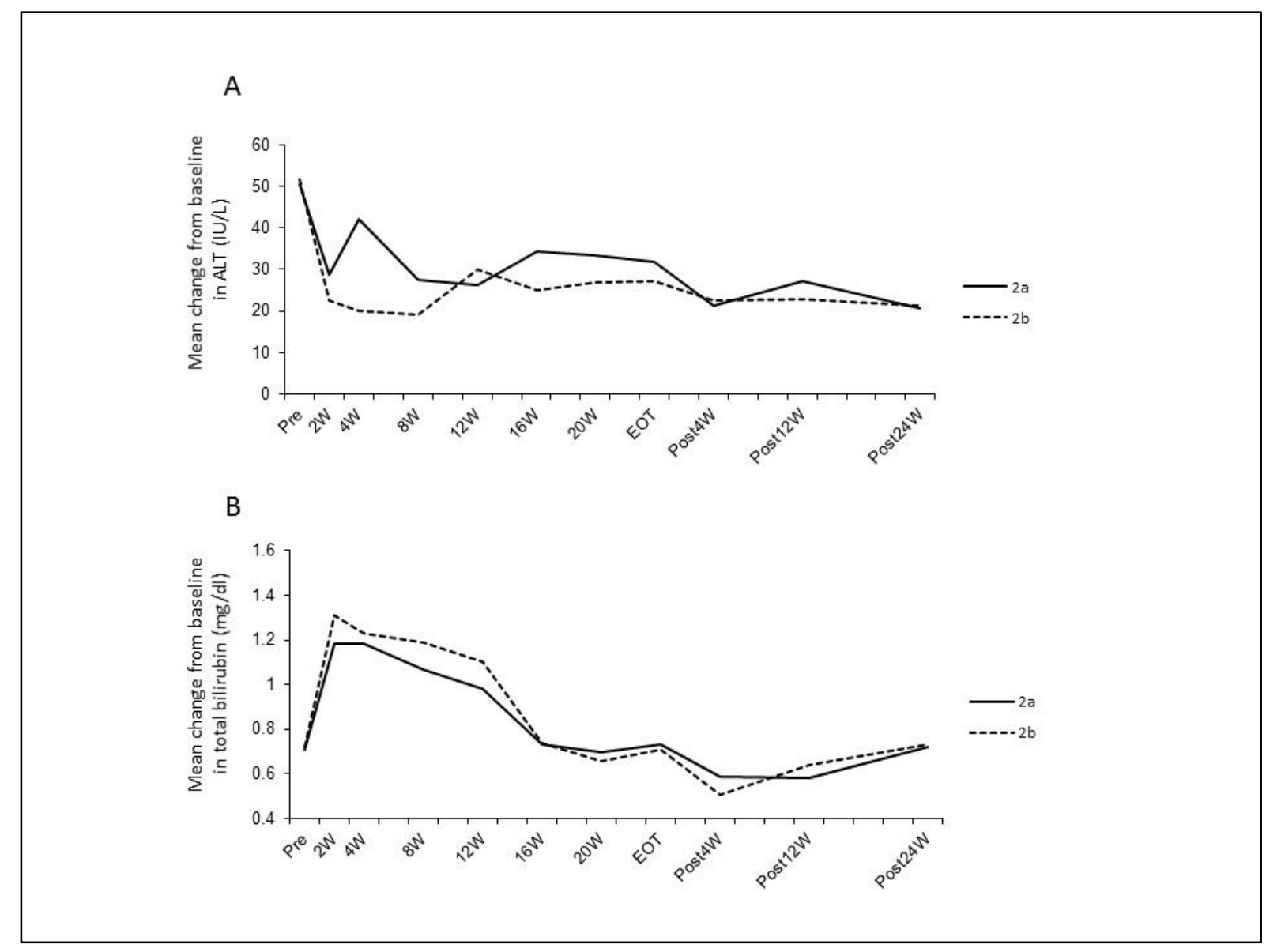


31

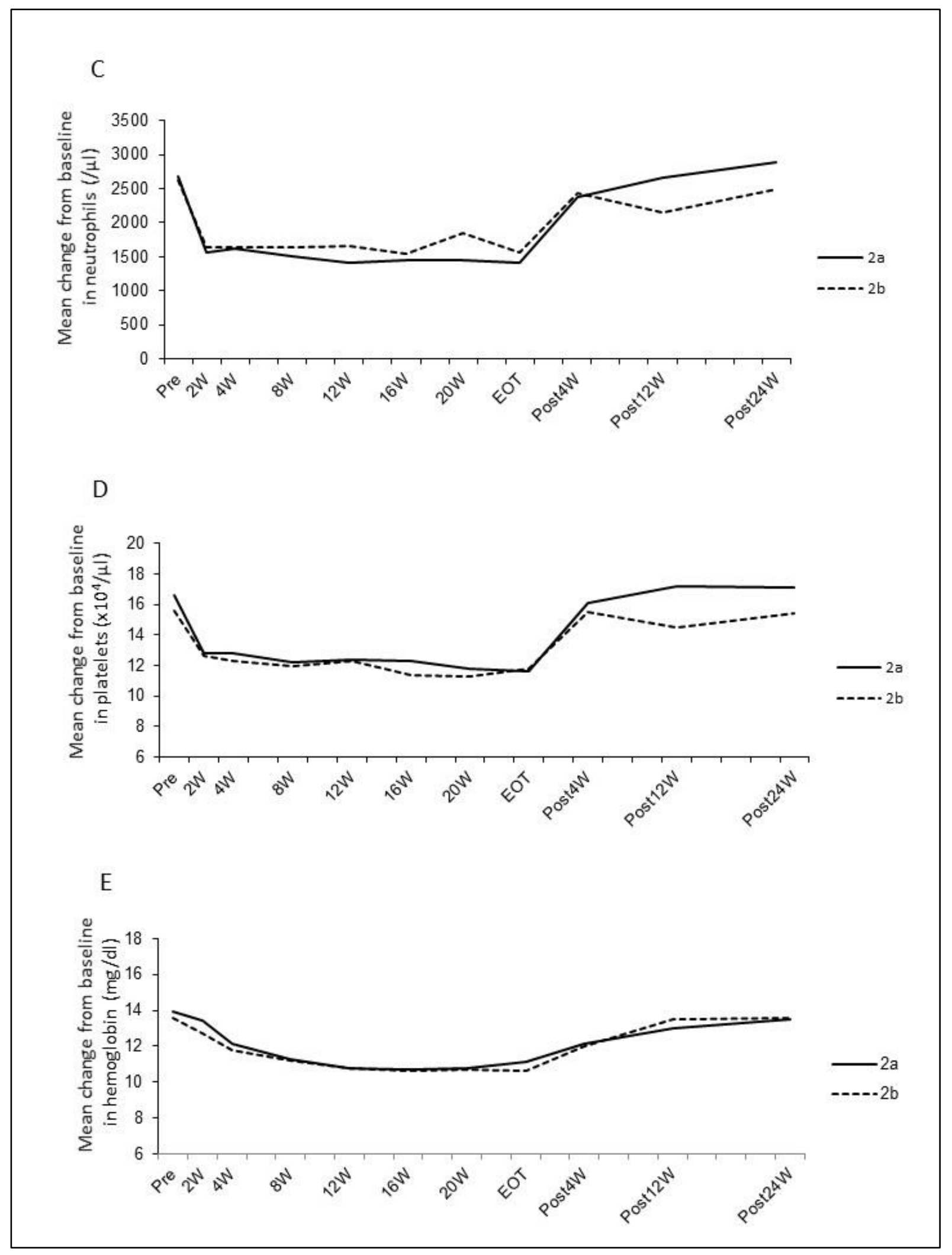

